\title{
Damage dependent stress limit model for failure prediction in bulk forming processes
}

\section{Journal Article}

\section{Author(s):}

Hora, Pavel; Tong, Longchang; Berisha, Bekim (D); Karadogan, Celalettin

Publication date:

2011

Permanent link:

https://doi.org/10.3929/ethz-b-000422754

Rights / license:

In Copyright - Non-Commercial Use Permitted

Originally published in:

International Journal of Material Forming 4(3), https://doi.org/10.1007/s12289-010-1002-y 


\title{
Damage dependent stress limit model for failure prediction in bulk forming processes
}

\author{
Pavel Hora $\cdot$ Longchang Tong $\cdot$ Bekim Berisha $\cdot$ \\ Celalettin Karadogan
}

Received: 7 December 2009/Accepted: 21 September 2010/Published online: 20 October 2010

(C) Springer-Verlag France 2010

\begin{abstract}
Stress limit, combined with deformation and orientation dependent damages, is proposed as the criterion for failure prediction in bulk forming processes. Dedicatedly designed experiments are carried out to investigate this model. The results verify the existence of the stress limits. The value of stress limit can vary in different directions due to the material processing histories. The experiments revealed also strong kinematical hardening behaviours for the investigated material. The constitutive law has to include this effect to evaluate the stresses with sufficient precision.
\end{abstract}

Keywords Complex non-proportional loading $\cdot$ Failure prediction · Stress limit criterion · Damage

\section{Introduction}

Failure prediction possesses great significance for the forming industry because enormous costs can be saved if the failures are discovered and eliminated in the prototyping phase by means of the virtual tools. The finite element method (FEM) is well developed to simulate the forming processes and provides systematic information about the processes. Therefore, FEM is no doubt the most powerful tool for the failure prediction in forming processes.

\footnotetext{
P. Hora $\cdot$ L. Tong $(\triangle) \cdot$ B. Berisha $\cdot$ C. Karadogan Institute of Virtual Manufacturing, ETH Zurich, Tannenstr. 3,

8092 Zurich, Switzerland

e-mail: tong@ivp.mavt.ethz.ch

URL: http://www.ivp.ethz.ch

P. Hora

e-mail: phora@ivp.mavt.ethz.ch
}

Some types of failure like wrinkling or geometrical defects can be directly predicted using the animation of the simulation results, while other kinds of failures such as rupture have to be predicted by means of some criteria based on either theoretical consideration or experimental results.

The concept of FLC has been widely used as criterion for fracture in the FEM simulation of sheet forming processes. In contrast, the failure prediction for the bulk forming processes still remains as a large deficiency although many models have been proposed to handle this problem. Extremely large deformations and high hydrostatic pressure as well as the complex deformation history make the prediction remarkably more difficult than by sheet forming.

Most of the existing models used for the failure prediction in bulk forming processes are either empirical formulae or functions of a scalar damage factor [1]. The necking or rupture is predicted when a certain criterion is fulfilled or the damage factor reaches a critical value.

Early works from Lemaitre [2] used the concept of damage and modified the stresses according to the damage in the material. Gurson [3] introduced the concept of void and described rupture as the results of void coalescence. These models have been proposed for decades. But the successful applications are still very limited to special cases.

One of the widely used models for failure prediction in bulk forming is the Oyane model [4]. Successful predictions have been reported in many works, e. g. [5, 6]. On the other hand, the accuracy of the Oyane criterion is not sufficient if the identical critical values are applied for different forming cases. Furthermore, this model ignores the anisotropic properties of the materials. 
Johnson and Cook obtained diagrams as the criteria for different metals under different loading conditions based on the experiments and computation with continuum damage mechanics [7]. The diagram is developed as relation between effective plastic strain and the stress triaxiality using so called round notched bar (RNB), as shown in Fig. 1. The stress triaxility is defined as the ratio of mean stress and yield stress $\sigma_{m} / \sigma_{Y}$. This kind of diagrams is also called as failure loci and is used in some commercial FE packages [9].

In the previous work [10], a direction oriented damage model was presented and the stress limit was suggested as the criterion for the material failure prediction in the FE simulation of bulk forming processes. This model assumes that the damages are strongly oriented according to the plastic deformations. The material strength is not affected by the damages when the compressive stress is applied. As the tensile stress is applied, the strength of the material decreases due to those damages. As soon as the tensile stress exceeds a limit value, rupture happens and the material fails.

\section{Stress limit criterion}

Tensile stresses, accompanied with plastic deformation, are primarily the driving force for the ductile fractures. The rupture appears only when the tensile stresses exceed the limit value. Those stress limits are determined by the properties of the material as well as by the deformation.
Analytical prediction of the Johnson-Cook triaxiality diagram

The stress limit criterion by simple cases can be written as:

$\sigma_{m a j} \leq \sigma_{c r i t}$.

Generally a stress state for the simple cases $\sigma_{22}=\sigma_{33}=$ $\alpha \sigma_{11}$ and $\tau_{12}=\beta \sigma_{11}$ can be expressed as

$\sigma_{i j}=\left[\begin{array}{lll}1 & \beta & 0 \\ \beta & \alpha & 0 \\ 0 & 0 & \alpha\end{array}\right] \sigma_{11}$.

Under the simplified assumption of isotropic v. Mises yield locus the equivalent stress for the stress states is

$\sigma_{e q}=\sqrt{\frac{1}{2}\left[\left(\sigma_{11}-\sigma_{22}\right)^{2}+\left(\sigma_{22}-\sigma_{33}\right)^{2}+\left(\sigma_{33}-\sigma_{11}\right)^{2}\right]+3 \tau_{12}{ }^{2}}$.

\section{CASE 1: main stress load case}

For a triaxial load case $(\alpha \neq 0$ and $\beta=0)$, which can be experimentally achieved with notched specimens (Fig. 1), we obtain

$$
\begin{aligned}
\sigma_{e q} & =\left(\sqrt{\frac{1}{2}\left[(1-\alpha)^{2}+(\alpha-\alpha)^{2}+(\alpha-1)^{2}\right]}\right) \sigma_{1} \\
& =(1-\alpha) \sigma_{1}
\end{aligned}
$$

Fig. 1 Diagram for the ductile fracture $[7,8]$

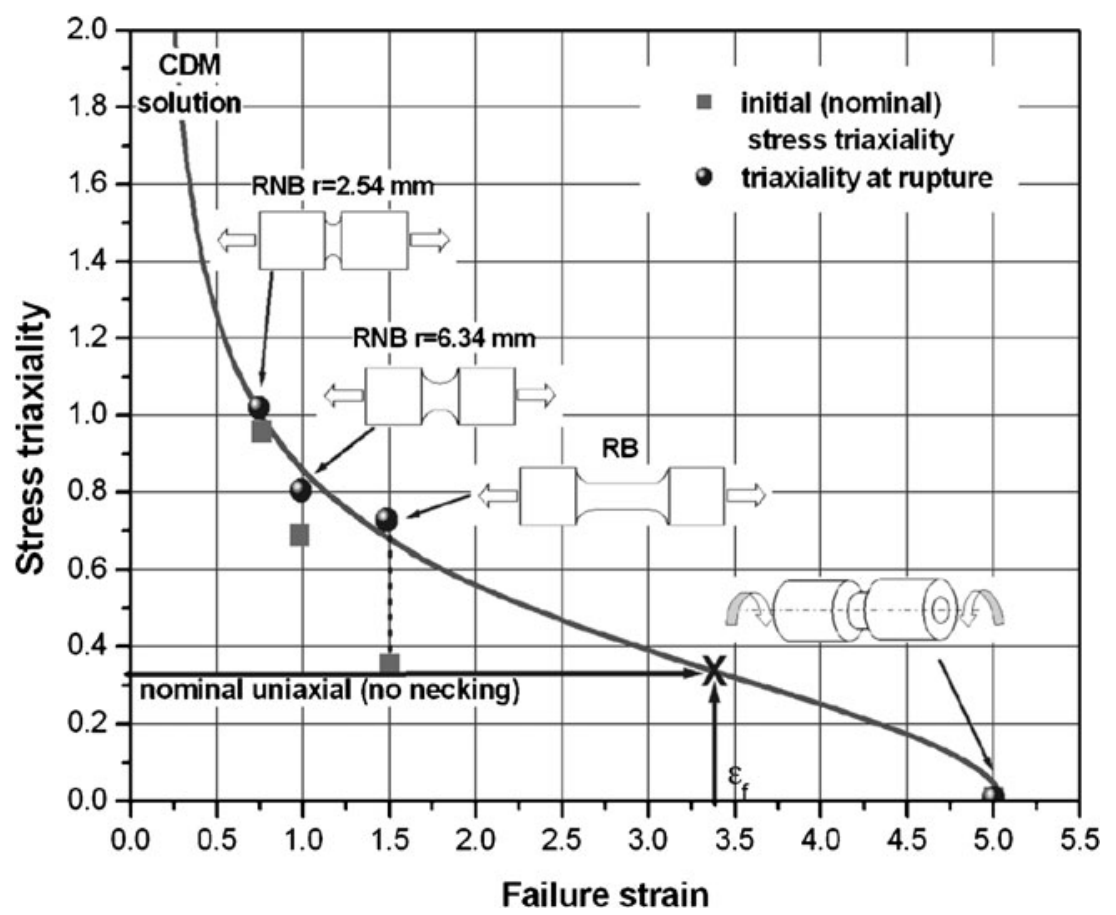


or

$\sigma_{1}(\alpha)=\frac{1}{(1-\alpha)} \sigma_{e q}$

If the hardening curve of a material is described using power law (Ludwik model) as

$\sigma_{Y}=A \varepsilon_{e q}^{n}$

and the material is under plastic state so that $\sigma_{e q}=\sigma_{Y}$, the major tensile stress is expressed as

$\sigma_{m a j}(\alpha)=\sigma_{Y} /(1-\alpha)=A \varepsilon_{e q}^{n} /(1-\alpha)$.

In the uniaxial tensile test where $\alpha=0$ the critical stress corresponds to

$\sigma_{\text {crit }}=A \varepsilon_{\text {crit_tensile }}^{n}$

For the general cases $\alpha \neq 0$ the stress limit model is expressed as

$\sigma_{m a j}(\alpha)=\sigma_{c r i t}$

Combination of (7), (8) and (9) results in the following relationship

$\varepsilon_{\text {crit_o }}=(1-\alpha)^{1 / n} \varepsilon_{\text {crit_tensile }}$

where the $\varepsilon_{\text {crit_ } \alpha}$ denotes the critical equivalent strain under triaxial loading conditions. For the case $\alpha \rightarrow 1$, we achieved the result $\varepsilon_{\text {crit }} \rightarrow 0$.

\section{CASE 2: pure torsion load}

By the torsion test the major stress is

$\sigma_{\text {torsion }}^{\text {major }}=\tau=\frac{\sigma_{Y}}{\sqrt{3}}=\frac{A}{\sqrt{3}}\left(\varepsilon_{\text {torsion }}\right)^{n}$.

Using the stress limit criterion, we arrive at the equation

$A\left(\varepsilon_{\text {crit_tensile }}\right)^{n}=\frac{A}{\sqrt{3}}\left(\varepsilon_{\text {crit_torsion }}\right)^{n}$.

After the simplification we obtain the relation

$\varepsilon_{\text {crit_torsion }}=(\sqrt{3})^{1 / n} \varepsilon_{\text {crit_tensile. }}$
For the value $n=0.36$ we get

$\varepsilon_{\text {crit_torsion }}=4.60 \varepsilon_{\text {crit_tensile }}$.

The much higher deformation achieved under pure torsion represents well the experimental evidence.

For more general hardening laws and anisotropic yield loci, as proposed for example by Barlat [11], the above stress-strain relations have to be evaluated numerically.

So long as the hardening curve and the stress limit for a material are available, the failure diagram can be evaluated for tension test of notched round bar as

$\Delta \varepsilon_{2}=\Delta \varepsilon_{3}=-0.5 \Delta \varepsilon_{1}$ and $\Delta \varepsilon_{1}=\Delta \varepsilon_{e q}$

Considering the rigid plastic material law $s_{1}=$ $\frac{2 \sigma_{Y}}{3 \Delta \varepsilon_{e q}} \Delta \varepsilon_{1}$, the major stress $\sigma_{1}$ is expressed as

$\sigma_{1}=s_{1}+\sigma_{m}=\frac{2}{3} \sigma_{Y}+\sigma_{m}=\frac{2}{3} \sigma_{Y}+\kappa \sigma_{Y} \leq \sigma_{c r i t}$

where $s_{1}$ denotes the major stress deviator and $\kappa=\frac{\sigma_{m}}{\sigma_{Y}}$ is the factor for stress triaxiality.

When the hardening curve is adopted as Johnson and Cook used in [7] for the material OFHC copper

$\sigma_{Y}=292 \varepsilon_{e q}^{0.31}+90$

and the stress limit is set as $550 \mathrm{MPa}$, the curve obtained using stress limit model is compared with the data provided by Johnson and Cook [7] in Fig. 2. The agreement is very well except for the value for pure torsion.

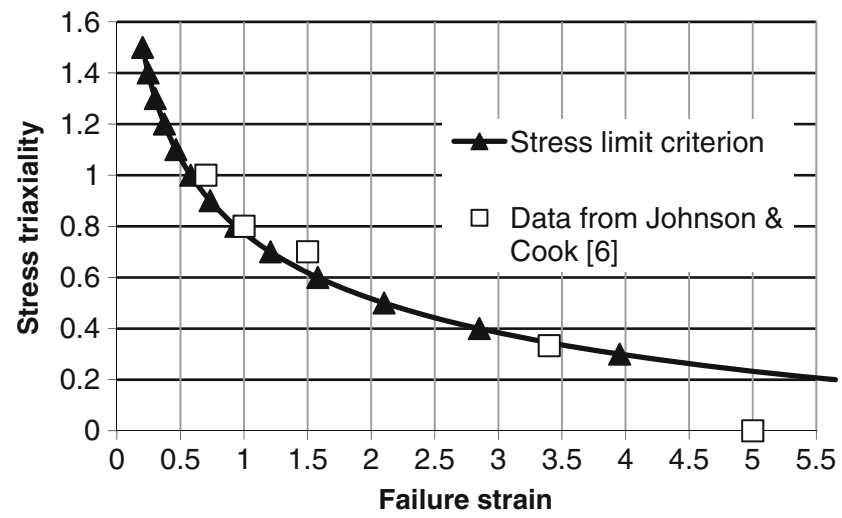

Fig. 2 Theoretical prediction of the critical strains in dependence of the stress triaxiality 
Fig. 3 Specimen for the compressiontensile test

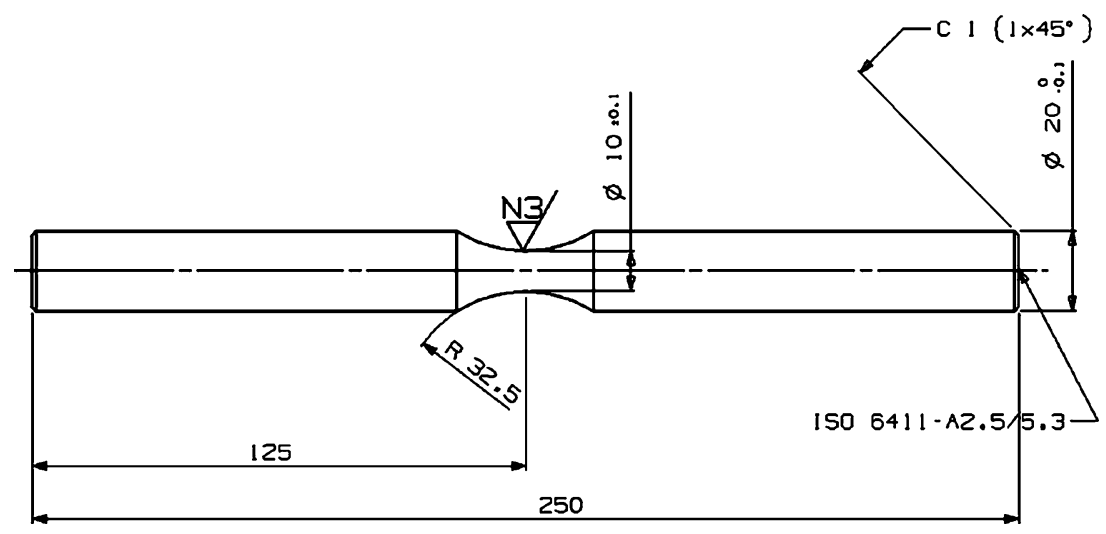

Stress triaxiality diagrams, however, may fail to predict failure properly in case of nonlinear loading paths. The OFHC copper mentioned above can be taken as example. Let the material be deformed under compressive condition at first to achieve the equivalent strain of 2.5. The corresponding equivalent stress at this intermediate state can be computed as $496 \mathrm{MPa}$ using Eq. 17. The critical triaxiality can be estimated as 0.46 with the diagram in Fig. 1. Consequently, critical mean stress $\sigma_{m}=\kappa \sigma_{Y}=228 \mathrm{MPa}$ is obtained. If the deformed material is loaded further under biaxial stretching state, problem arises using the triaxiality diagram. For biaxial stretching $\sigma_{1}=\sigma_{2}$ and $\sigma_{3}=0$ the mean stress is calculated as $\sigma_{m}=\frac{2}{3} \sigma_{1}$. It leads to the result $\sigma_{1}=\frac{3}{2} \sigma_{m}=342$ $\mathrm{MPa}$ using the critical value provided by the triaxiality diagram. If failure should be predicted for this state, it is obviously incorrect because the stress is still below the yield stress and the material is still in the elastic state. As soon as the material is plastified, that means $\sigma_{1}=\sigma_{Y}$, the triaxiality value reaches immediately the level of 0.666 , which lies far above the curve. In contrast, the stress limit criterion predicts failure when the stretching stress exceeds the critical value of $550 \mathrm{MPa}$. Therefore further plastic deformation is necessary to cause the material failure.

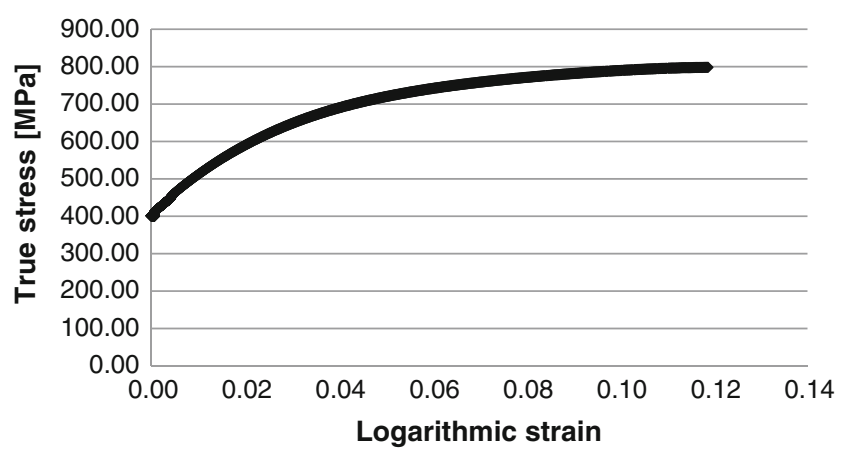

Fig. 4 Hardening curve of the steel SAE 1144
Modelling of deformation state dependent damages

Experiments showed that the damages inside the material are strongly orientation dependent. A specimen that

a

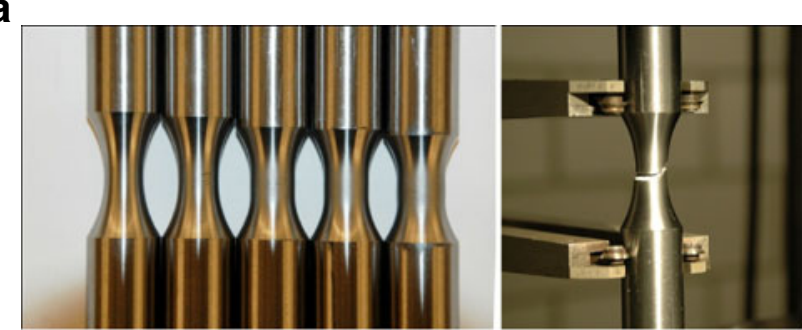

b 0.6

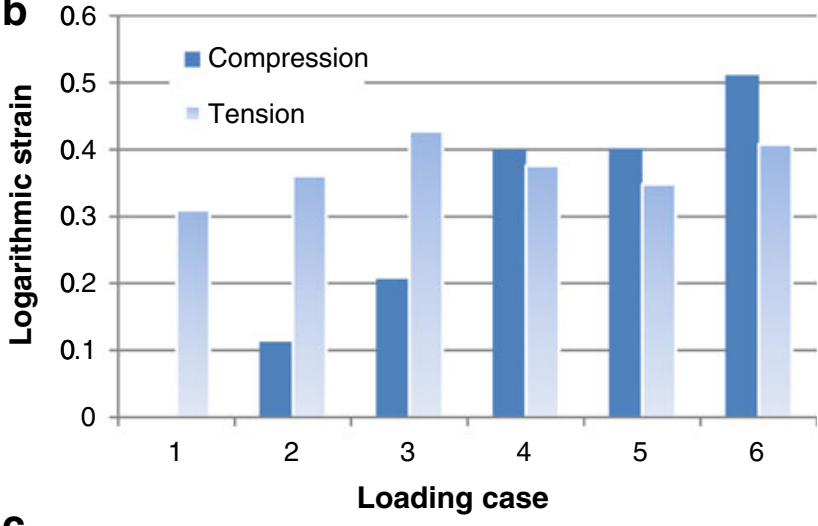

C

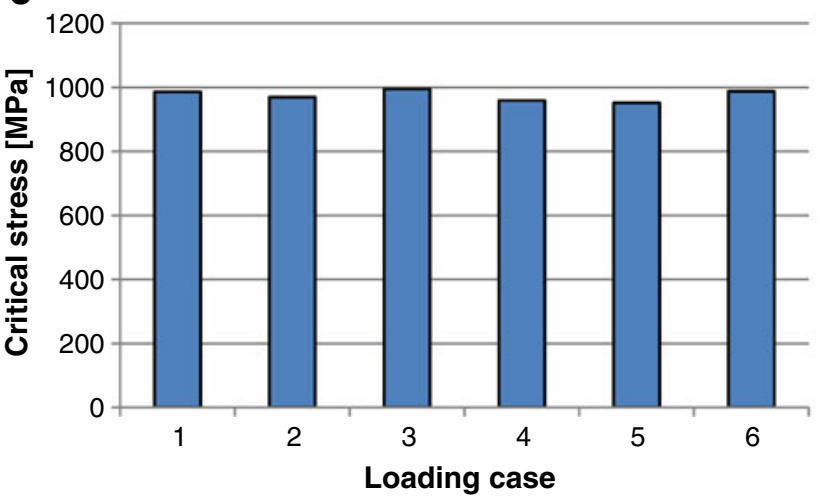

Fig. 5 Experimental evaluation of critical stresses by compressiontensile test 
Table 1 Compression-tensile test

\begin{tabular}{|c|c|c|c|c|c|c|c|}
\hline Nr. & $\mathrm{D}_{0}[\mathrm{~mm}]$ & $\mathrm{D}_{\text {compression }}[\mathrm{mm}]$ & $\varepsilon_{\text {comp }}$ & $\mathrm{D}_{\text {tensile }}[\mathrm{mm}]$ & $\varepsilon_{\text {tensile }}$ & Maximum tensile force $(\mathrm{kN})$ & Stress limit $[\mathrm{MPa}]$ \\
\hline 1 & 10.03 & 10.03 & 0 & 8.59 & 0.310 & 57.16 & 986 \\
\hline 2 & 10.02 & 10.6 & 0.113 & 8.85 & 0.361 & 59.65 & 970 \\
\hline 3 & 10.03 & 11.12 & 0.206 & 8.89 & 0.448 & 63.02 & 995 \\
\hline 4 & 10.03 & 12.25 & 0.400 & 10.15 & 0.376 & 77.6 & 959 \\
\hline 5 & 10.03 & 12.26 & 0.401 & 10.3 & 0.348 & 79.3 & 952 \\
\hline 6 & 10.03 & 12.95 & 0.511 & 10.56 & 0.408 & 86.48 & 987 \\
\hline
\end{tabular}

undergoes compressive deformation can be further compressed without noticeable damage. The rupture might appear much earlier as the loading direction changes. In order to consider the influence of deformation history, the damage dependent stress limit model is presented.

This model is based on the assumption that the plastic deformations cause material damages. Such damages weaken the strength of the material consequently. It is then reasonable to define the damage $D$ as an objective function of the deformation gradient tensor $\mathbf{F}$

$D=f(\mathbf{F})$.

An infinitesimal material section area $\mathrm{d} \mathbf{A}_{0}$ is transformed to $\mathbf{d A}$ with the deformation gradient tensor as:

$\mathrm{d} \mathbf{A}=(\operatorname{det} \mathbf{F})\left(\mathbf{F}^{-1}\right)^{T} \mathrm{~d} \mathbf{A}_{0}$.

If the damage is induced by the deformation, the difference of the real section area and ideal section area can be defined as the expression of damage

$D=\frac{\left(d A^{\text {ideal }}-d A^{\text {real }}\right)}{d A^{\text {ideal }}}$.

Unlike the existing models where a scalar value is adopted for the damage description, we use 3 values to distinguish the damages in 3 principal direction of the deformation tensor:

$D_{i}=\left\{\begin{array}{ll}0 & \left(\left|\varepsilon_{i}\right| \leq \varepsilon_{0}\right) \\ \Gamma\left(\left|\varepsilon_{i}\right|-\varepsilon_{0}\right) & \left(\left|\varepsilon_{i}\right|>\varepsilon_{0}\right)\end{array} \quad(i=1,2,3)\right.$

where $\varepsilon_{0}$ denotes the threshold strains for the initialization of damages in each direction. Here the function $\Gamma$ describes the relation between damage and plastic strain. In the work of Lemaitre [2], a model was established for isotropic materials in the frame of Continuous Damage Mechanics (CDM). In the same work he verified also the linearity of the function between damages and plastic strain using 6 different metal materials as experimental samples. However, the choice of functions is not unique and the parameters of the functions must be well calibrated using the experimental results.

In the case of non-proportional loading case, (21) loses the validity because the principal directions are changing and the description for damage rate and strain rate has to be adopted. Further investigations are needed therefore for the general cases.
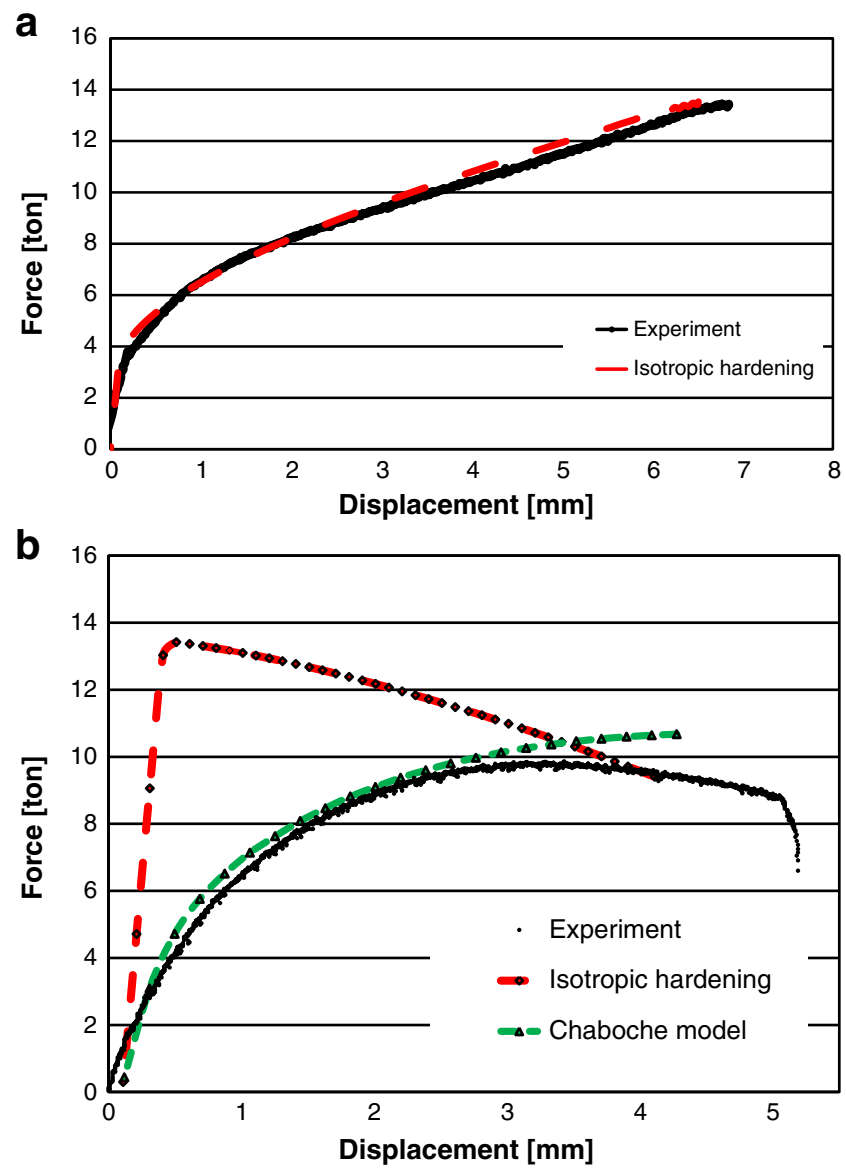

Fig. 6 Force-Displacement curves for the compression-tensile test. a) Good agreement between the simulation and experiment; b) Deviations under the assumption of isotropic hardening and the improvement due to Chaboche model 
Table 2 Parameters of Chaboche model for material SAE 1144

\begin{tabular}{llllllll}
\hline $\mathrm{C}_{1}$ & $\mathrm{C}_{2}$ & $\mathrm{C}_{3}$ & $\eta_{1}$ & $\eta_{2}$ & $\mathrm{~b}$ & $\mathrm{Q}_{0}$ & $\mathrm{Q}_{\infty}$ \\
\hline 1264.43 & 3724.54 & 22.49 & 19.35 & 50.19 & 22.04 & 494.15 & 308.46 \\
\hline
\end{tabular}

3D stress limit criterion (3D-SLC)

Since damages exist in the material, the stresses calculated with an ideal material model are obviously lower than the stresses in real cases. The existing models intend to modify the material constitutive laws using the damage factor. However, as the damages do not affect the material properties under compressive stress states, it is not necessarily favorable to do this modification at the level of constitutive relation.

As an alternative method to include the effect of damages, we introduce the limit tensile stress as the critical value. The stress limit is reduced by damages in the material. Because only tensile stresses are compared with the critical values, it is no more necessary to change the constitutive relations. Mathematically, the critical stresses in different directions can be calculated with the corresponding damages

$\sigma_{i b}^{*}=\sigma_{i b}\left(1-D_{i}\right) \quad(i=1,2,3)$.

The initial stress limits in different directions form a spherical surface for isotropic materials without damages. However, many engineering raw materials have been processed with different methods such as rolling, drawing or heat treatment before they are put into the current bulk forming processes. If the material possesses anisotropic properties, or the initial damages are direction dependent, the stress limits might be different in different directions. The locus of stress limits will form then an ellipsoid surface. Mathematically the criterion can be expressed as:

$\left(\frac{\sigma_{n} n_{1}}{\sigma^{*}{ }_{1 b}}\right)^{2}+\left(\frac{\sigma_{n} n_{2}}{\sigma^{*}{ }_{2 b}}\right)^{2}+\left(\frac{\sigma_{n} n_{3}}{\sigma^{*}{ }_{3 b}}\right)^{2} \leq 1$.

Here $\sigma_{n}$ is the normal stress along an arbitrary direction and $n_{1}, n_{2}$ and $n_{3}$ are the direction cosines of this direction.
Experimental evaluation of the critical stress

In order to verify the model and estimate the stress limit of steel SAE 1144, compression-tensile tests were performed. The geometry of the specimen is shown in Fig. 3. The hardening curve of this steel is obtained using standard tensile test as shown in Fig. 4. The specimens were compressed to different amounts to achieve compressive deformation. Afterwards the specimens were deformed under tensile condition until rupture appeared (Fig. 5a).

During the experiment, the force and displacement of the process are recorded and the diameters in the middle of the specimens are measured before and after the deformations (Table 1). The deformations are calculated using logarithmic strain as

$$
\begin{aligned}
\varepsilon_{\text {comp }} & =\ln \left(D_{\text {compression }}^{2} / D_{0}^{2}\right) \text { and } \varepsilon_{\text {tensile }} \\
& =\ln \left(D_{\text {tensile }}^{2} / D_{\text {compression }}^{2}\right)
\end{aligned}
$$

and the critical stresses are calculated with a mean value as

$\sigma^{\text {crit }}=F_{\max } /\left(\pi D_{\text {tensile }}^{2} / 4\right)$.

Despite the remarkable different deformation histories (Fig. 5b), the critical stresses obtained from the tensile test show only slight variation, as shown in Fig. $5 \mathrm{c}$.

Experimental data can be used for the determination of parameters for the model. As the data in Table 1 are used for the mathematical approximation approach, the values for (21) are obtained as $\varepsilon_{0}=0.004$ and $\Gamma=0.07$ for the linear relation $D=\Gamma \cdot\left(\varepsilon-\varepsilon_{0}\right)$ between damage and plastic strain, while the stress limit of initial material in (22) is also obtained as $\sigma_{i b}=1050[\mathrm{MPa}]$. The small value of $\Gamma$ indicates a slow accumulation process of damage in this

Fig. 7 The bending and rebending test
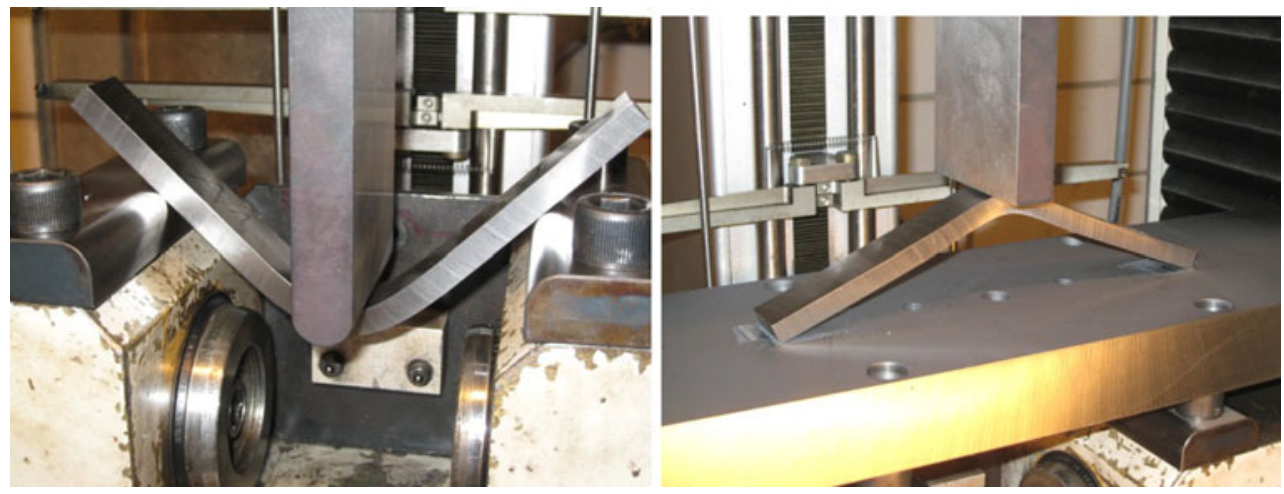
Fig. 8 The one way bending test and the simulation, critical stress at failure
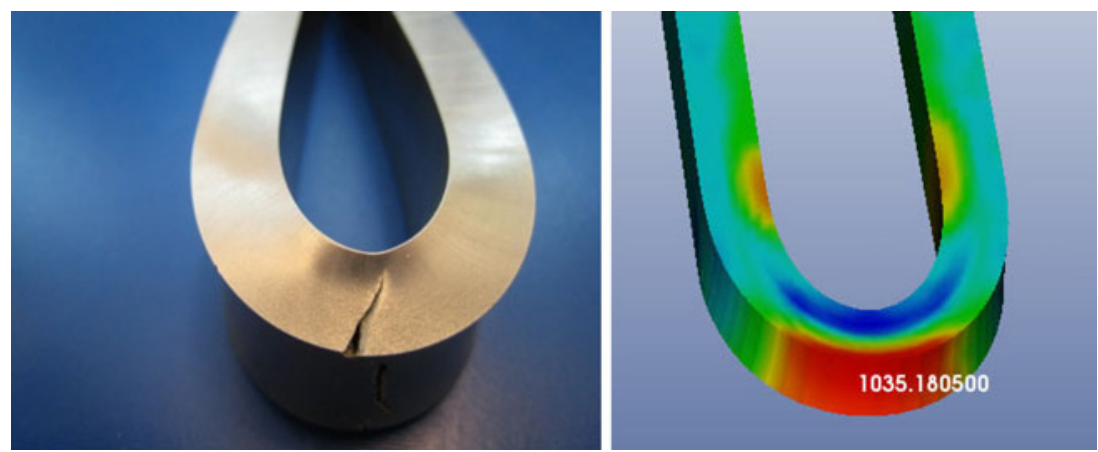

material. That provides the explanation why the stress limit is not strongly affected by the plastic deformations.

However, as only very limited experimental data are available for the approximation, the significance of the approximation results is also limited. More experiments should be performed and more complex stress states should be investigated in order to obtain more accurate description of the damage accumulation. Nevertheless, the experiment confirmed the feasibility of using critical stress as failure criterion.

\section{Kinematic hardening}

Since the stress is adopted as the criterion for the failure prediction, the precision of the evaluation of stresses becomes essential in the computation.

Many metal materials show the Bauschinger effect. If non-proportional loading is applied, the isotropic hardening model is unable to describe the material behaviors properly.

As an example, the above compression-tensile test is computed using the isotropic hardening model. Excellent agreement between experiment and calculation was obtained by the simulation of compression process (Fig. 6a). However, as the loading changed to the tensile, the isotropic model overestimated the load (Fig. 6b).

Therefore, the Chaboche model [12] for the kinematical hardening is introduced. This model describes the material behavior well when the loading direction changes, as shown in Fig. 6b.

Back stresses are introduced to describe the behavior of kinematical hardening. The yield function can be expressed as:

$f=J_{2}(\sigma-\mathbf{X})-Q \leq 0$

where $J_{2}$ is the second invariant of the stress deviator, $\boldsymbol{X}$ is the back-stress and $Q$ describes the strain hardening effect of the material. The evolution equation of the back-stress proposed by Chaboche was

$\mathbf{d} \mathbf{X}_{i}=\frac{2}{3} C_{i} \mathbf{d} \varepsilon^{p}-\eta_{i} \mathbf{X}_{i} d \varepsilon_{e q}$

where $C_{i}$ and $\eta_{i}$ are material constants which describe translation of the yield surface in the stress space. In order to describe complex material behavior, the back-stress is defined as the sum of nonlinear as well as of linear components

$\mathbf{X}=\sum_{i=1}^{n} \mathbf{X}_{i}$

The model uses a common exponential function $Q$ to describe the strain hardening behavior. $Q$ consists of a strain independent yield stress $Q_{0}$, a saturation value $Q_{\infty}$ and a saturation rate $b$

$$
Q=Q_{0}+Q_{\infty}\left(1-\exp \left(-b \varepsilon_{e q}\right)\right)
$$

Fig. 9 Comparison of calculated stresses at the end of first bending of $90^{\circ}$

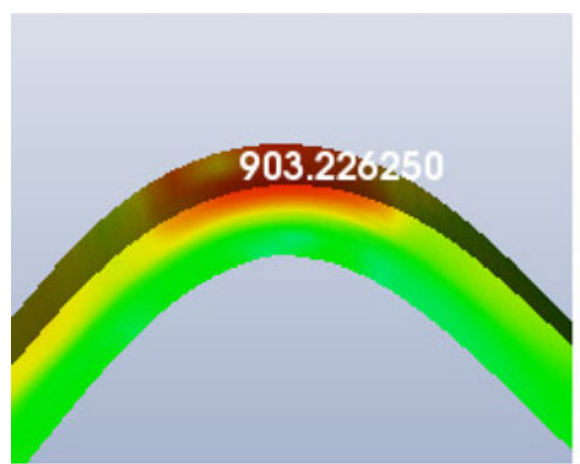

a Isotropic hardening

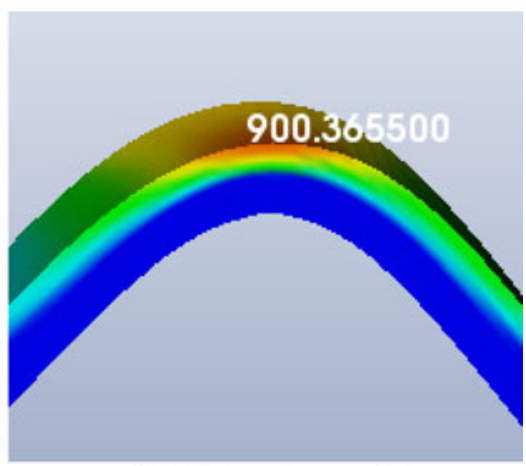

b Chaboche model 
Fig. 10 Results with different models a) failure predicted by isotropic model, b) no failure predicted by Chaboche model

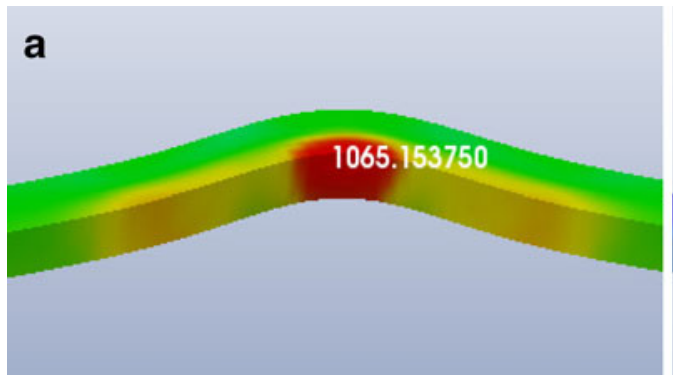

b

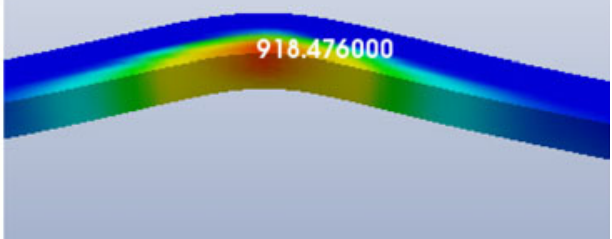

In order to determine the parameters for the Chaboche model, experiments were carried out and the parameters are determined with the experimental data [13]. The parameters used in this work are listed in Table 2.

\section{Experimental verification}

Several experiments have been performed in order to verify the stress limit model. In this work the bending experiment is presented as well.

A bar was bended with the equipment shown in Fig. 7. Two different loading cases were performed. In the first case, the bar was bended in just one direction until the fracture appeared in the middle. In the second case the specimen was bended to around $90^{\circ}$ at first. It was then bended in the reverse direction until fracture appeared.

Both processes are calculated using isotropic hardening and Chaboche model. In the one way bending simulation (Fig. 8), both models worked very well and we obtained the critical tensile stress for the investigated material as $1035 \mathrm{MPa}$ by comparing the simulations and experiment.

In the multi-step bending no fracture appeared at the end of the first pre-bending of $90^{\circ}$. The simulation of both models showed the same results as in Fig. 9.

The difference of the two models appeared as they were used to simulate the subsequent reverse bending processes. Because isotropic hardening model doesn't take the Bauschinger effect into account, it delivered overestimated values for stresses. Failure should be predicted as the specimen was bended to near flat form because the tensile stress exceeded the limit value (Fig. 10a). However, in the experiment, no fracture was observed at this stage. In contrast, Chaboche model described the material behavior properly. The maximum stress value was below the critical stress of $1035 \mathrm{MPa}$.

By the further bending process the tensile stress with Chaboche model reached the critical value. Fig. 11 shows the comparison of the simulation and the specimen. Very satisfactory agreement is achieved by the Chaboche model.

\section{Conclusions and outlook}

The stress limit can be well used as the criterion for the rupture prediction in bulk forming processes. In order to achieve sufficient accuracy, kinematical hardening has to be included in the constitutive relation for many materials with strong Bauschinger effect. Moreover, large plastic deformations in bulk forming processes cause damages in the materials and the stress limit can be direction and damage dependent. The implementation into an FE program delivers a convenient judgment for the possible failure.

However, the prediction of ductile fracture in forming process is a very challenging theme. Besides the deformation history and stress states, more factors such as strain rate and temperature might also play important roles in the failure process. Different metals or different processing histories can
Fig. 11 Result with Chaboche model and rupture by experiment
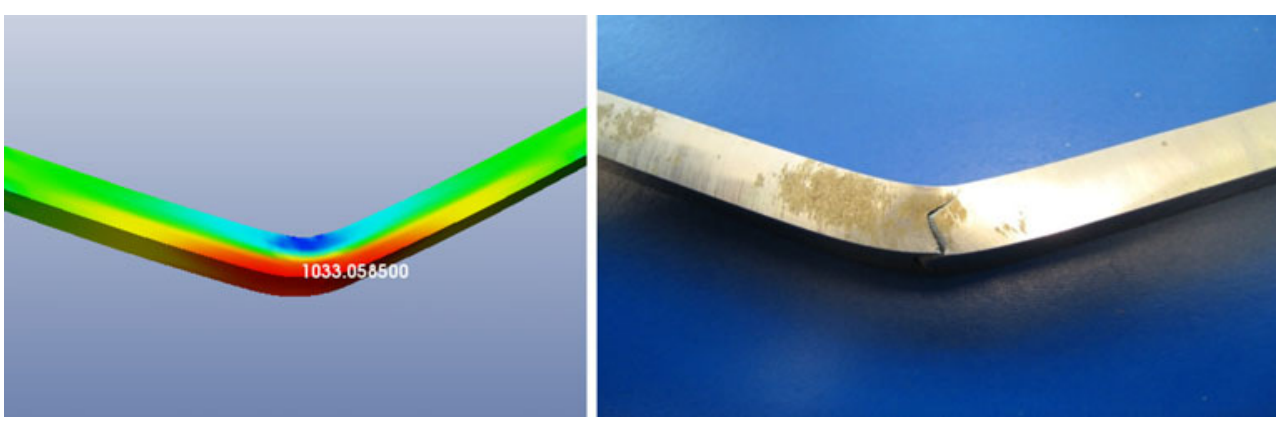
also lead to different rupture types. Therefore, more experiments with different materials under complex forming conditions have to be carried out in the future investigations to supply the model with systematical experimental data.

\section{References}

1. Lin J, Liu Y, Dean TA (2005) A review on damage mechanisms, models and calibration methods under various deformation conditions. Int J Damage Mech 14:299-319

2. Lemaitre JA (1985) A continuous damage mechanics model for ductile fracture. J Eng Mater Tech 107:83-89

3. Gurson A (1977) Continuum theory of ductile rupture by void nucleation and growth, Part I: yield criteria and flow rules for porous ductile materials. J Eng Mater Tech 99:2-15

4. Oyane $M$ et al (1979) Criteria for ductile fracture and their applications. J Mech Work Tech 4:65-81

5. Gouveia B et al (1996) Fracture predicting in bulk forming. Int J Mech Sci 38(4):361-372
6. McAllen P et al (2005) A method for the prediction of ductile fracture by central bursts in axisymmetric extrusion. Mech Eng Sci Part C 219:237-250

7. Johnson GR, Cook WH (1985) Fracture characteristics of three metals subjected to various strains, strain rates, temperatures and pressures. Eng Fract Mech 21:31-48

8. Bonora N, Gentile D, Pirondi A (2004) Identification of the parameters of a non-linear continuum damage mechanics model for ductile failure in metals. J Strain Analysis 39(6):639-651

9. Abaqus Analysis User's manual (2007) Damage initiation for ductile metals, section 19.2.2 Version 6.7

10. Hora P, Tong L, Berisha B (2007) Stress limit model with deformation dependent damage for failure prediction in bulk forming processes, NumiForm 2007, Porto Portugal, pp. 1401-1406

11. Barlat $\mathrm{F}$ et al (2005) Linear transformation-based anisotropic yield functions. Int J Plast 21:1009-1039

12. Chaboche JL (1989) Constitutive equations for cyclic plasticity and cyclic viscoplasticity. Int J Plast 5:247-302

13. Berisha B et al (2008) Modeling of dynamic strain aging with a dislocation-based isotropic hardening model and investigation of orthogonal loading. In: 9th International Conference on Technology of Plasticity, pp. $185-186$ 